\title{
One-Pot Facile Synthesis of Acridinediones and their Derivatives by Nano Ferrite as a Catalyst
}

\author{
JHANSI RANI SUNKARA ${ }^{1 *}$, MURALIKRISHNA RALLABHANDI ${ }^{1}$, \\ SRIDHAR PRASANGI ${ }^{2}$ and MAHESH PALLA ${ }^{1 *}$ \\ ${ }^{1}$ Department of PNCO, Andhra University, Visakhapatnam-530003, India \\ ${ }^{2}$ Optimus drugs Ltd., Hyderabad \\ mahi143vzm@gmail.com
}

Received 19 May 2016 / Accepted 4 June 2016

\begin{abstract}
The synthesis of 9-aryl substituted acridinedione derivatives was achieved by employing nano ferrite catalyst under solvent free conditions. XRD and TEM results exhibits cubic phase of maghemite $\left(\gamma-\mathrm{Fe}_{2} \mathrm{O}_{3}\right)$ with average particle size of $20-25 \mathrm{~nm}$. Catalytic activity of the nano ferrite was explored for the synthesis of acridinedione. The present approach offers several advantages such as short reaction time, easy purification, high yield and reusability of the catalyst.
\end{abstract}

Keywords: Nano ferrite, Heterogeneous catalyst, Acridines, Solvent free, Reusability

\section{Introduction}

Acridine derivatives have a wide spectrum of biological activities such as antibacterial, anti-cancer $^{1}$, anti-malarial ${ }^{2}$, antifungal ${ }^{3}$, anti-tumor ${ }^{4}$ and cytotoxic activities. For example Amsacrine is an antineoplastic agent. Natural, synthetic acridines and their derivatives are effective DNA and RNA-binding compounds owing to their intercalation abilities as well as being a lipophilic carrier molecule ${ }^{5,6}$. Synthesis of Acridinediones is a continuing focal point of current research because these moieties are active pharmaceutical ingredients (API's) and also valuable reactive intermediates for both synthetic and medicinal chemists ${ }^{7}$.

Literature survey reveals that various methods ${ }^{8}$ have been reported for preparation of acridinediones and substituted acridinediones. The reported method (Scheme 1) for the synthesis of 3,4,6,7-tetrahydro-9-phenylacridine-1,8 $(2 \mathrm{H}, 5 \mathrm{H}, 9 \mathrm{H}, 10 \mathrm{H})$-dione involves the reaction of two molecules of dimedone (1,3-cyclohexane) with various aromatic aldehydes and ammonium acetate by using different Lewis acid catalysts ${ }^{9}$. However many of these catalysts undergo disadvantages such as long reaction time, high catalyst loading, use of solvents and deactivation of catalyst on repeated use. Hence there is a need to develop an environmentally benign protocol for the synthesis of acridinediones. 
Though there are many advantages of homogeneous metals catalysts there are some difficulties in recovering the catalyst from the reaction mixture, which severely inhibit their use in industry. Heterogeneous catalyst effects in easy separation and recycling of nano catalyst. Recent reports showed that magnetic nano particles are efficient catalysts and they can be easily separated from the reaction mixture ${ }^{10}$. The high surface to volume ratio of metal oxide nano particles is mainly responsible for their high catalytic performance. Ferrite nano material is one such reusable catalyst which shows profound catalytic activity in organic synthesis. Nano ferrite is a non-hygroscopic, inexpensive, non-toxic material, which has been utilized as a heterogeneous catalyst for various organic reactions. This method offers advantageous such as short reaction time, recyclability of the catalyst and easy work-up procedure.
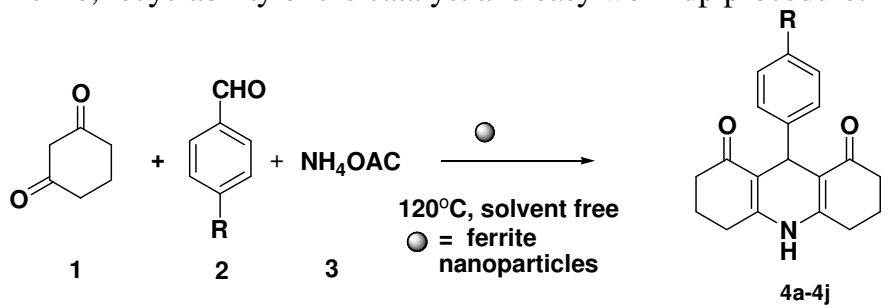

$\mathrm{R}: \mathrm{a}=\mathrm{H} ; \mathrm{b}=-3,4\left(\mathrm{OCH}_{3}\right)_{2} ; \mathrm{c}=-3,4,5\left(\mathrm{OCH}_{3}\right)_{3} ; \mathrm{d}=-\mathrm{N}\left(\mathrm{CH}_{3}\right)_{2} ; \mathrm{e}=-(\mathrm{OH})\left(\mathrm{OCH}_{3}\right) ; \mathrm{f}-\mathrm{N}\left(\mathrm{Et}_{2}\right)$; $\mathrm{g}=-(\mathrm{OH}) ; \mathrm{h}=-\mathrm{p}-\mathrm{OCH}_{3} ; \mathrm{i}=-\mathrm{F} ; \mathrm{j}=-2,6(\mathrm{di} \mathrm{Cl})$

Scheme 1. Synthesis of 3,4,6,7-tetrahydro-9-phenylacridine-1,8(2H,5H,9H,10H)-dione employing reusable nano ferrite as catalyst

In this study, we report a simple, efficient, and one-pot reaction of 1, 3 cyclohexanedione, aldehydes and ammonium acetate using nano ferrite at $120{ }^{\circ} \mathrm{C}$ for the preparation of acridinediones and their derivatives.

\section{Experimental}

1, 3-Diketones and aldehydes were obtained from Aldrich chemicals. Melting points were determined on a Buchi 504 apparatus and were uncorrected. IR spectra were recorded in $\mathrm{KBr}$ pellets on a Nicolet (Impact 410) FT-IR spectrophotometer. ${ }^{1} \mathrm{H}$ NMR and ${ }^{13} \mathrm{C}$ NMR spectra were recorded on a Varian Mercury Plus $400 \mathrm{MHz}$ NMR spectrophotometer using tetramethylsilane (TMS) as an internal standard. Coupling constants are expressed in hertz. The progress of the reaction was monitored by thin layer chromatography (TLC) that runs on silica gel G (Merck).

\section{Preparation of nano-ferrite particles}

$\mathrm{Fe}\left(\mathrm{NO}_{3}\right)_{3} \cdot 9 \mathrm{H}_{2} \mathrm{O}$, polyethylene glycol (PEG2000) and potassium chloride $(\mathrm{KCl})$ were of analytical grade. Double distilled water was used in preparation. The preparation procedure was as follows: Initially, $0.006 \mathrm{~mol} \mathrm{Fe}\left(\mathrm{NO}_{3}\right)_{3} \cdot 9 \mathrm{H}_{2} \mathrm{O}$ was dissolved in $100 \mathrm{~mL}$ aqueous solution of PEG2000 with a $\mathrm{NO}_{3} / \mathrm{PEG} 2000$ molar ratio of 1 . Then, the desired amount of $\mathrm{KCl}$ was added to the above solution and the resulting transparent solution was thoroughly stirred by a magnetic mixer at $70{ }^{\circ} \mathrm{C}$ until a homogeneous sol-like solution was observed. Afterwards, the sol-like solution was dried at $100^{\circ} \mathrm{C}$ for $24 \mathrm{~h}$. The obtained gel was placed in a silica crucible and heated in air until the gel was ignited. The ash-burned powder was boiled in deionized water to remove the salt. Final product obtained after filtering was washed with deionized water and ethanol, then dried ${ }^{11}$ at $80{ }^{\circ} \mathrm{C}$ for $2 \mathrm{~h}$.

General synthesis of 3, 4, 6, 7-tetrahydro-9-phenylacridine-1, $8(2 \mathrm{H}, 5 \mathrm{H}, 9 \mathrm{H}, 10 \mathrm{H})$-dione

A mixture of 1,3cyclohexanedione (2 eq) (1), substituted aromatic aldehydes (1 eq) (2), Ammonium acetate (3) and $\mathrm{Fe}_{2} \mathrm{O}_{3}$ nano particles $(15 \mathrm{~mol} \%)$ were stirred at $120{ }^{\circ} \mathrm{C}$ in an oil 
bath for the prescribed time. The completion of the reaction was monitored by TLC. After completion of the reaction, the reaction was cooled to room temperature to attain a solid product. Then the product was dissolved in ethanol and the catalyst was recovered by magnetization. The crude products were further purified by recrystallization from ethanol. All the synthesized products were characterized by IR, NMR and Mass spectroscopic data and their melting points were compared with authentic samples.

\section{Results and Discussion}

Synthesis of 3, 4, 6, 7-tetrahydro-9-phenylacridine-1,8(2H,5H,9H,10H)-dione using Nano ferrite

Initially, a blank reaction was performed using benzaldehyde, cyclohexanedione and ammonium acetate (mole rate $1: 2: 1$ ) at $120{ }^{\circ} \mathrm{C}$ in the absence of nano ferrite to establish the efficacy of the catalyst and the results showed that desired product was not formed even after 12 hours of heating. Then focus was diverted to optimize amount of catalyst and solvent effect. In order to evaluate the most appropriate catalyst percentage, a model reaction using benzaldehyde, ammonium acetate and 1,3 cyclohexanedione (mole ratio1:1:2) was carried out using 0-20 mol\% of nano ferrite under solvent-free conditions (Table 1).

Table 1. Optimization of the molar ratio of the of the catalyst for the model reaction at $120{ }^{\circ} \mathrm{C}$

\begin{tabular}{cccc}
\hline Product & Catalyst mole \% & Yield & Time, min \\
\hline & 0 & 0 & $12, \mathrm{~h}$ \\
& 10 & 96 & 8 \\
& 15 & 96 & 6 \\
& 20 & 94 & 7
\end{tabular}

It was found that $10 \mathrm{~mol} \%$ of nano ferrite showed high yield in short reaction time at $120{ }^{\circ} \mathrm{C}$ (Table 1). During our ongoing studies, effect of solvent was studied for the above model reaction with $10 \mathrm{~mol} \%$ of catalyst (Table 2). Ultimately solvent free reactions were preferable in terms of excellent yield in short reaction times.

Table 2. Effect of solvent on the sample reaction

\begin{tabular}{ccccc}
\hline Entry & Solvent & Amount of catalyst, mol \% & Time, $\mathrm{h}$ & Yield, \% \\
\hline 1 & Acetonitrile & 10 & 2.0 & 59 \\
2 & THF & 10 & 3.0 & 46 \\
3 & Ethanol & 10 & 3.0 & 71 \\
4 & 1,4-Dioxane & 10 & 4.0 & 57 \\
5 & Methanol & 10 & 3.0 & 56 \\
6 & Water & 10 & 4.0 & 41 \\
7 & Neat & 10 & $10(\mathrm{~min})$ & 95 \\
\hline
\end{tabular}

The catalyst was recovered by magnetization after completion of the reaction, washed with diethyl ether and the recovered catalyst was reused for four cycles. During washing with the solvent, it was observed that there was no leaching of catalyst and was confirmed by performing the reaction with the filtrate. From our investigations, we observe that nano catalyst shows excellent to good reactivity with promising yields even for the next four cycles in the same reaction (Table 3 ). 
Table 3. Reusability of nano catalyst

\begin{tabular}{cccc}
\hline S.No & Catalyst & Recovery\% & Yield \\
\hline 1 & - & 96 \\
2 & 96 & 88 \\
3 & 87 & 81 \\
4 & 81 & 79 \\
\hline
\end{tabular}

Under the optimized conditions, aromatic aldehyde (4a-4j) containing electron donating as well as electron withdrawing groups with different substitution patterns were effectively cyclized to give 3, 4, 6,7-tetrahydro-9-phenylacridine-1,8 $(2 \mathrm{H}, 5 \mathrm{H}, 9 \mathrm{H}, 10 \mathrm{H})$-dione (Table 4).

Table 4. One-pot synthesis of acridines catalyzed by Ferrite nanoparticals

\begin{tabular}{|c|c|c|c|c|c|}
\hline Entry & Aldehyde & Nitrogen Source & Product & Time, min & Yield, \% \\
\hline 1 & & $\mathrm{NH}_{4} \mathrm{OAC}$ & $4 \mathbf{a}$ & 6 & 88 \\
\hline 2 & & $\mathrm{NH}_{4} \mathrm{OAC}$ & $4 b$ & 7 & 92 \\
\hline 3 & & $\mathrm{NH}_{4} \mathrm{OAC}$ & $4 c$ & 8 & 93 \\
\hline 4 & & $\mathrm{NH}_{4} \mathrm{OAC}$ & 4d & 7 & 97 \\
\hline 5 & & $\mathrm{NH}_{4} \mathrm{OAC}$ & $4 e$ & 7 & 96 \\
\hline 6 & & $\mathrm{NH}_{4} \mathrm{OAC}$ & $4 f$ & 8 & 96 \\
\hline 7 & & $\mathrm{NH}_{4} \mathrm{OAC}$ & $4 g$ & 8 & 98 \\
\hline 8 & & $\mathrm{NH}_{4} \mathrm{OAC}$ & $4 h$ & 8 & 94 \\
\hline 9 & & $\mathrm{NH}_{4} \mathrm{OAC}$ & $4 i$ & 6 & 95 \\
\hline 10 & & $\mathrm{NH}_{4} \mathrm{OAC}$ & $4 \mathbf{j}$ & 6 & 97 \\
\hline
\end{tabular}

All the products were identified with m.p., ${ }^{1} \mathrm{H} N M R .{ }^{13} \mathrm{C}$ NMR and FT-IR Spectroscopy techniques. The spectral data is given below 


\section{Spectral data}

3,4,6,7-Tetrahydro-9-phenylacridine-1, $8(2 \mathrm{H}, 5 \mathrm{H}, 9 \mathrm{H}, 10 \mathrm{H})$-dione (4a)

M.p 269-272 ${ }^{0} \mathrm{C},{ }^{1} \mathrm{H}$ NMR (400 MHz, DMSO, $\delta$, ppm): 1.693-1.769 (m, 2H, $1 \mathrm{CH}_{2}$ ), 1.786$1.886\left(\mathrm{~m}, 2 \mathrm{H}, 1 \mathrm{CH}_{2}\right), 2.189\left(\mathrm{~s}, 8 \mathrm{H}, 4 \mathrm{CH}_{2}\right), 4.896(\mathrm{~s}, 1 \mathrm{H}, \mathrm{CH}), 9.419(\mathrm{~s}, 1 \mathrm{H}, \mathrm{NH}) ;{ }^{13} \mathrm{CNMR}(100$

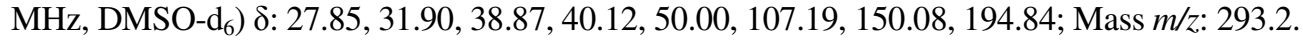

3,4,6,7-Tetrahydro-9-(3,4-dimethoxyphenyl)acridine-1,8(2H, 5H, 9H, 10H)-dione (4b)

M.P $255-258{ }^{0} \mathrm{C},{ }^{1} \mathrm{H}$ NMR (400MHz, DMSO, $\delta$, ppm): 1.753-1.891(m, 4H, 2CH $), 1.903-$ 1.912(t, $\left.2 \mathrm{H}, \mathrm{CH}_{2}\right), 1.912-1.924\left(\mathrm{t}, 2 \mathrm{H}, \mathrm{CH}_{2}\right), 2.185-2.198\left(\mathrm{t}, 2 \mathrm{H}, \mathrm{CH}_{2}\right), 2.198-2.217\left(\mathrm{t}, 2 \mathrm{H}, \mathrm{CH}_{2}\right)$, $3.647\left(\mathrm{~s}, 6 \mathrm{H}, 2 \mathrm{OCH}_{3}\right), 4.849(\mathrm{~s}, 1 \mathrm{H}, \mathrm{CH}), 6.584(\mathrm{~s}, 1 \mathrm{H}, \mathrm{Ar}-\mathrm{H}), 6.729(\mathrm{~s}, 1 \mathrm{H}, \mathrm{Ar}-\mathrm{H}), 6.769$ (s, $1 \mathrm{H}, \mathrm{Ar}-\mathrm{H}), 9.379(\mathrm{~s}, 1 \mathrm{H}, \mathrm{NH}) ;{ }^{13} \mathrm{CNMR}\left(100 \mathrm{MHz}, \mathrm{DMSO}-\mathrm{d}_{6}\right) \delta: 28.7,31.8,39.9,40.1,50.0$, 55.6, 112.5, 114.6, 114.9, 120.2, 135.3, 144.8, 146.8, 162.5, 195.9; Mass $m / z$ : 354.1[M+1].

3,4,6,7-Tetrahydro-9-(3,4,5-trimethoxyphenyl)acridine-1,8(2H, 5H, 9H, 10H)-dione (4c)

M.P $279-282{ }^{0} \mathrm{C},{ }^{1} \mathrm{H}$ NMR (400MHz, DMSO, $\delta$, ppm): $1.795-1.935\left(\mathrm{~m}, 4 \mathrm{H}, 2 \mathrm{CH}_{2}\right), 2.217-$ $2.240\left(\mathrm{t}, 4 \mathrm{H}, 2 \mathrm{CH}_{2}\right), 2.491-2.516\left(\mathrm{t}, 4 \mathrm{H}, 2 \mathrm{CH}_{2}\right), 3.657\left(\mathrm{~s}, 3 \mathrm{H}, \mathrm{OCH}_{3}\right), 3.657\left(\mathrm{~s}, 6 \mathrm{H}, 2 \mathrm{OCH}_{3}\right.$ ),4.894 (s, 1H, CH), 6.410 (s, 2H, 2 Ar-H), 9.422 (s, 1H, NH); Mass m/z: 383.2.

3,4,6,7-Tetrahydro-9-(4-(dimethyl amino)phenyl)acridine-1,8(2H, 5H, 9H, 10H)-dione (4d)

M.p 164-166 ${ }^{0} \mathrm{C},{ }^{1} \mathrm{H}$ NMR (400 MHz, DMSO, $\delta$, ppm, 1.747-1.819 (m, 2H, $\mathrm{CH}_{2}$ ), 1.876$1.920\left(\mathrm{~m}, 2 \mathrm{H}, \mathrm{CH}_{2}\right), 2.143-2.182\left(\mathrm{t}, 2 \mathrm{H}, \mathrm{CH}_{2}\right), 2.313-2.346\left(\mathrm{t}, 2 \mathrm{H}, \mathrm{CH}_{2}\right), 2.478-2.500(\mathrm{t}, 2 \mathrm{H}$, $\left.\mathrm{CH}_{2}\right), 2.815\left(\mathrm{~s}, 6 \mathrm{H}, 2 \mathrm{CH}_{3}\right), 4.784(\mathrm{~s}, 1 \mathrm{H}, \mathrm{CH}), 6.516--6.537$ (d, 1H, Ar-H), 6.575--6.596 (d, $1 \mathrm{H}, \mathrm{Ar}-\mathrm{H}), 6.847--6.868$ (d, 1H, Ar-H), 6.929--6.950 (d, 1H, Ar-H), $9.332(\mathrm{~s}, 1 \mathrm{H}, \mathrm{NH}) ;{ }^{13} \mathrm{C}$ NMR $\left(100 \mathrm{MHz}\right.$, DMSO-d $\left.{ }_{6}\right) \delta:$ 20.34, 20.82, 26.28, 30.76, 36.81, 40.36, 112.21, 112.37, 112.96, 126.81, 127.96, 135.83, 148.55,150.63,194.72; Mass $m / z: 336.8$.

3,4,6,7-tetrahydro-9-(4-hydroxy-3-methoxyphenyl) acridine-1, $8(2 \mathrm{H}, 5 \mathrm{H}, 9 \mathrm{H}, 10 \mathrm{H})$ dione (4e)

M.p 268-271 ${ }^{0} \mathrm{C},{ }^{1} \mathrm{H}$ NMR (400 MHz, DMSO, $\delta$, ppm): 1.783-1.886 (m, 2H, $\mathrm{CH}_{2}$ ), 1.897$1.930\left(\mathrm{~m}, 2 \mathrm{H}, \mathrm{CH}_{2}\right), 2.192-2.205\left(\mathrm{t}, 4 \mathrm{H}, 2 \mathrm{CH}_{2}\right), 2.211-2.501\left(\mathrm{t}, 4 \mathrm{H}, 2 \mathrm{CH}_{2}\right), 3.669(\mathrm{~s}, 3 \mathrm{H}$, $\left.\mathrm{OCH}_{3}\right), 4.826(\mathrm{~s}, 1 \mathrm{H}, \mathrm{CH}), 6.452-6.476(\mathrm{~d}, 1 \mathrm{H}, \mathrm{Ar}-\mathrm{H}), 6.538-6.559(\mathrm{~d}, 1 \mathrm{H}, \mathrm{Ar}-\mathrm{H}), 6.732-6.736$ $(\mathrm{d}, 1 \mathrm{H}, \mathrm{Ar}-\mathrm{H}), 8.569$ (s, 1H, OH), $9.370(\mathrm{~s}, 1 \mathrm{H}, \mathrm{NH}) ;{ }^{13} \mathrm{CNMR}\left(100 \mathrm{MHz}, \mathrm{DMSO}-\mathrm{d}_{6}\right) \delta: 20.4$, 29.6, 36.5, 40.5, 56.2, 111.9, 115.6, 122.7, 130.9,148.4,149.5, 198.9; Mass $m / z: 339.1$.

3,4,6,7-Tetrahydro-9-(4-(diethyl amino)phenyl)acridine-1,8(2H, 5H, 9H, 10H)dione $(4 \mathrm{f})$

M.p 255-257 ${ }^{0} \mathrm{C},{ }^{1} \mathrm{H}$ NMR (400 MHz, DMSO, $\delta$, ppm, 1.003-1.038(t,6H,2CH$)$, 1.754-1.804 (m, 2H, $\mathrm{CH}_{2}$ ), 1.879-1.921 (m, 2H, $\left.\mathrm{CH}_{2}\right), 2.172-2.195$ (t, $\left.4 \mathrm{H}, 2 \mathrm{CH}_{2}\right), 2.465-2.501$ (t, $4 \mathrm{H}$, $\left.2 \mathrm{CH}_{2}\right), 3.201-3.253\left(\mathrm{q}, 4 \mathrm{H}, 2 \mathrm{CH}_{2}\right), 4.762(\mathrm{~s}, 1 \mathrm{H}, \mathrm{CH}), 6.420-6.442(\mathrm{~d}, 2 \mathrm{H}, 2 \mathrm{Ar}-\mathrm{H}), 6.89-$ $6.912(\mathrm{~d}, 2 \mathrm{H}, 2 \mathrm{Ar}-\mathrm{H}), 9.333(\mathrm{~s}, 1 \mathrm{H}, \mathrm{NH}) ;{ }^{13} \mathrm{C}$ NMR (100 MHz, DMSO-d 6 ) $\delta: 12.51,20.81$, 26.29, 30.60, 36.84, 43.51, 111.00, 113.04, 128.15, 134.44, 145.38, 150.61, 194.77; Mass $m / z: 365.8[\mathrm{M}+1]$.

3,4,6,7-tetrahydro-9-(2-hydroxy phenyl)acridine-1,8(2H, 5H, 9H, 10H)-dione (4g)

M.p 267-269 ${ }^{0} \mathrm{C}$, ${ }^{1} \mathrm{H}$ NMR (400 MHz, DMSO, $\delta$, ppm, 1.792-1.837 (m,2 $\left.\mathrm{H}_{1} \mathrm{CH}_{2}\right)$, 1.913$1.958\left(\mathrm{~m}, 2 \mathrm{H}, \mathrm{CH}_{2}\right), 2.252-2.299\left(\mathrm{t}, 4 \mathrm{H}, 2 \mathrm{CH}_{2}\right), 2.556-2.586\left(\mathrm{t}, 4 \mathrm{H}, 2 \mathrm{CH}_{2}\right), 4.870(\mathrm{~s}, \mathrm{H}, \mathrm{CH})$, 6.662-6.684 (d, 2H, Ar-H), 6.803-6.807(d, H, Ar-H), 6.949-6.953 (d, H, Ar-H), 9.684 (s, H, $\mathrm{NH}), 9.813$ (s, H, OH); Mass $m / z: 310.1[\mathrm{M}+1]$. 
3,4,6,7-Tetrahydro-9-(4-methoxyphenyl)acridine-1,8(2H, 5H, 9H, 10H)-dione (4h)

M.p 195-198 ${ }^{0} \mathrm{C},{ }^{1} \mathrm{H}$ NMR (400 MHz, DMSO, $\delta$, ppm): 1.75-1.783 (m, 2H, $\mathrm{CH}_{2}$ ), 1.878$1.923\left(\mathrm{~m}, 2 \mathrm{H}, \mathrm{CH}_{2}\right), 2.176-2.202\left(\mathrm{t}, 4 \mathrm{H}, 2 \mathrm{CH}_{2}\right), 2.498-2.507\left(\mathrm{t}, 4 \mathrm{H}, 2 \mathrm{CH}_{2}\right), 3.658(\mathrm{~s}, 3 \mathrm{H}$, $\left.\mathrm{OCH}_{3}\right), 4.837$ (s, 1H,CH), 6.701-6.723 (d, 2H, Ar-H), 7.027-7.048 (d, 2H, Ar-H), 9.402 (s, $1 \mathrm{H}, \mathrm{NH}) ;{ }^{13} \mathrm{C}$ NMR $\left(100 \mathrm{MHz}, \mathrm{DMSO}-\mathrm{d}_{6}\right) \delta: 20.80,26.29,31.15,36.78,54.85,112.71$, 113.10, 128.35, 139.67, 150.98, 157.11, 194.76; Mass $m / z: 323.5$

3,4,6,7-Tetrahydro-9-(4-fluorophenyl)acridine-1,8(2H, 5H, 9H, 10H)-dione (4i)

M.p 270-272 ${ }^{\circ} \mathrm{C},{ }^{1} \mathrm{H}$ NMR (400 MHz, DMSO, $\delta$, ppm): 1.759-1.792 (m, 2H, $\mathrm{CH}_{2}$ ), 1.8451.926(m, $\left.2 \mathrm{H}, \mathrm{CH}_{2}\right), 2.186-2.212\left(\mathrm{t}, 4 \mathrm{H}, 2 \mathrm{CH}_{2}\right), 2.499-2.512\left(\mathrm{t}, 4 \mathrm{H}, 2 \mathrm{CH}_{2}\right), \quad 4.89$ (s, $1 \mathrm{H}, \mathrm{CH}), 6.966(\mathrm{~s}, 2 \mathrm{H}, \mathrm{Ar}-\mathrm{H}), 7.156(\mathrm{~s}, 2 \mathrm{H}, \mathrm{Ar}-\mathrm{H}), 9.49(\mathrm{~s}, 1 \mathrm{H}, \mathrm{NH}) ;{ }^{13} \mathrm{CNMR}(100 \mathrm{MHz}$, DMSO-d 6 ) $\delta:{ }^{13} \mathrm{C}$ NMR (100 MHz, DMSO-d 6 ) $\delta: 26.42,28.97,32.04,32.60,50.12,110.99$, 127.43, 129.38, 129.87, 146.02, 149.42, 194.24;

\section{3,4,6,7-tetrahydro-9-(2,6-di chloro phenyl)acridine-1,8(2H, 5H, 9H, 10H)-dione (4j)}

M.p 246-249 ${ }^{\circ} \mathrm{C},{ }^{1} \mathrm{H}$ NMR (400 MHz, DMSO, $\delta$, ppm): 1.728-1.875(m,4H,2 $\left.\mathrm{CH}_{2}\right), 2.011-$ $2.070\left(\mathrm{t}, 4 \mathrm{H}, 2 \mathrm{CH}_{2}\right), 2.097-2.15\left(\mathrm{t}, 4 \mathrm{H}, 2 \mathrm{CH}_{2}\right), 5.536(\mathrm{~s}, \mathrm{H}, 2 \mathrm{CH}), 7.039-7.059(\mathrm{~d}, \mathrm{H}, \mathrm{Ar}-\mathrm{H})$, 7.221-7.250(d, H, Ar-H), 7.460-7.480 (d, H, Ar-H), 9.464 (s, H, NH); Mass m/z: 391.4

The $\mathrm{Fe}_{2} \mathrm{O}_{3}$ nano particle catalyst plays a crucial role in the success rate of the reaction. In the absence of the $\mathrm{Fe}_{2} \mathrm{O}_{3}$ nano particles the reaction of 1,3-cyclohexanediones (1) and benzaldehyde derivative (2) could be carried out, but no product was obtained, but in the presence of catalyst, product was formed within a short reaction time. Since metal oxide play the role as a heterogeneous catalyst in many chemical industries and the rate of reaction on the catalyst surface depends on the total surface area and the number of active sites on the catalyst, a good catalytic activity was obtained from smaller partial size and high surface area of the catalyst. The crucial role of $\mathrm{Fe}_{2} \mathrm{O}_{3}$ nano particles as a good catalyst was obviously revealed.

\section{Conclusion}

A convenient and highly efficient method for the synthesis of 3,4,6,7-tetrahydro-9phenylacridine- $1,8(2 \mathrm{H}, 5 \mathrm{H}, 9 \mathrm{H}, 10 \mathrm{H})$-dione by condensation reaction of aldehydes with 1,3 cyclohexane-diones by the use of nano- $\mathrm{Fe}_{2} \mathrm{O}_{3}$ as an inexpensive and easily recoverable catalyst has been described. The attractive features of this synthetic protocol are easy work up procedure, operational simplicity and reusability of the catalyst. Furthermore, products were isolated in good to excellent yields.

\section{Acknowledgement}

The authors are thankfull to BSR fellow ship for providing financial assistance. The authors are also grateful to Andhra University, Vishakhapatnam.

\section{References}

1. (a) Gamage S A, Spicer J A, Atwell G J, Finlay G J, Baguley B C and Denny W A, J Med Chem., 1999, 42(13), 2383-2393; DOI:10.1021/jm980687m (b) Pitta M G R, Souza E S, Barros F W A, Filho M O M, Pessoa O, Hernandes M Z, Lima M D C A D, Galdino S L and Pitta I D R, Med Chem Res., 2013, 22(5), 2421-2429; DOI:10.1007/s00044-012-0236-2 (c) Venkatesan K, Pujari S S and Srinivasan K V, Synth Commun., 2008, 39(2), 228-241; DOI:10.1080/00397910802044306 
2. Girault S, Grellier P, Berecibar A, Louis M, Elisabeth M, Pascal L, Marie A D, Elisabeth D C and Christian S, J Med Chem., 2000, 43(14), 2646- 2654; DOI:10.1021/jm990946n

3. Srivastava A and Nizamuddin C, Indian J Heterocycl Chem., 2004, 13, 261-264.

4. Mikata Y, Yokoyama M, Mogami K, Kato M, Okura I, Chikira M and Yano S, Inorg Chim Acta, 1998, 279(1), 51-57; DOI:10.1016/S0020-1693(98)00035-8

5. Topcu Z, J Clin Pharm Ther., 2001, 26(6), 405-416; DOI:10.1046/j.13652710.2001.00368.x

6. Van Mouwerik T J, Caines P M and Ballentine R, Drug Intell Clin Pharm., 1987, 21(4), 330-334.

7. Heravi M M, Bakhtiari K, Zadsirjan V, Bamoharram F F, Heravi O M, Bioorg Med Chem., 2007, 17(15), 4262-4265; DOI:10.1016/j.bmcl.2007.05.023

8. Smolders R R, Waefelaer A, Coomans R, Francart D, Hanuise J and Nvoglet N, Bull Soc Chim Belg., 1982, 91, 33-42.

9. Kidwai $\mathrm{M}$ and Bhatnagar D, Tetrahedron Lett., 2010, 51(20), 2700-2703; DOI:10.1016/j.tetlet.2010.03.033

10. Velu R, Malar E J P, Ramakrishna V T and Ramamurthy P, Tetrahedron Lett., 2010, 51, 5680-5685; DOI:10.1016/j.tetlet.2010.08.042

11. Mahesh P, Guruswamy K, Diwakar B S, Rama Devi B, Murthy Y L N, PratapK and Pammi S V N, Chem Lett., 2015, 44(10), 1386-1388; DOI:10.1246/cl.150503 